\title{
Utilizacão da Transferência de Embrião em equinos no município de Mojuí dos Campos, no Baixo Amazonas, Pará
}

\section{Use of Embryo Transfer in horses in the municipality of Mojuí dos Campos, in Baixo Amazonas, Pará}

\author{
Jâmily Katrine Bezerra Freire Neves ${ }^{1}$, Gabrielle Medeiros Zanin ${ }^{1 *}$, Kedson Alessandri Lobo \\ Neves ${ }^{2}$, Leticia dos Santos Rebelo ${ }^{1}$, Helder Ribeiro Batista ${ }^{1}$, Welligton Conceição da Silva ${ }^{3}$, \\ Raimundo Nonato Colares Camargo Júnior ${ }^{4}$
}

\begin{abstract}
RESUMO
A técnica de transferência de embrião possibilita a evolução no desenvolvimento do setor no mercado equino através do aumento da eficiência reprodutiva e no progresso do melhoramento genético das raças e seus cruzamentos, sendo uma importante biotécnica que permite a potencialização genética dos animais, aumentando o número de produtos obtidos por égua ao ano. O objetivo deste estudo foi observar a quantidade de embriões coletados e o número de prenhezes confirmadas aplicando-se a técnica de transferência de embrião no município de Mojuí dos Campos, na mesorregião do Baixo Amazonas, Pará. Para isto, foram utilizadas 5 éguas doadoras e 25 éguas receptoras para se coletar embriões, durante o período de um ano. Os animais foram acompanhados por meio de um aparelho ultrassonográfico por via transretal, obtendo informações do crescimento folicular e tônus do útero. Os folículos foram medidos individualmente para o controle de sincronização entre doadora e receptoras. Como resultado, foram coletados 10 embriões, sendo as doadoras D2 e D4 as com maior número de coletas, especificamente, quatro e três coletas. Os embriões coletados foram transferidos para as receptoras, totalizando 10 transferências de embriões. Destas, $80 \%$ apresentaram prenhez positiva aos 15 dias e mantiveram esse resultado por ocasião da confirmação da gestação, aos 25 dias, levando a prenhez até o nascimento do potro.
\end{abstract}

Palavras-chave: Doadora; Lavagens uterinas transcervicais; Receptora; Coleta de embrião.

\begin{abstract}
The embryo transfer technique enables the evolution of the sector's development in the equine market through increased reproductive efficiency and progress of genetic improvement of breeds and their crossings, being an important biotechnique that allows the genetic enhancement of animals by increasing the number of products obtained per mare per year. The aim of this study was to observe the number of embryos collected and the number of confirmed pregnancies by applying the embryo transfer technique in the municipality of Mojuí dos Campos, in the mesoregion of Baixo Amazonas, Pará. For this, 5 donor mares were used. and 25 recipient mares to collect embryos, over a period of one year. The animals were followed
\end{abstract}

\footnotetext{
${ }^{1}$ Centro Universitário da Amazônia (UNAMA).

* E-mail: gabrielle.zanin18@gmail.com

${ }^{2}$ Universidade Federal do Oeste do Pará (UFOPA)

${ }^{3}$ Universidade Federal Rural da Amazônia (UFRA)

${ }^{4}$ Instituto Federal de Educação, Ciência e Tecnologia do Pará (IFPA)
} 
up using a transrectal ultrasound device, obtaining information on follicular growth and uterine tone. Follicles were individually measured to control synchronization between donor and recipient. As a result, 10 embryos were collected, being the D2 and D4 donors the ones with the highest number of collections, specifically, four and three collections. The collected embryos were transferred to the recipients, totaling 10 embryo transfers. Of these, $80 \%$ had a positive pregnancy at 15 days and maintained this result at the time of pregnancy confirmation, at 25 days, leading to pregnancy until the foal's birth.

Keywords: Donor; Transcervical uterine washings; Receiver; Embryo collect.

\section{INTRODUÇÃO}

A extensão de novas técnicas reprodutivas possibilitou o melhor aproveitamento dos animais, tornando possível acelerar o melhoramento das linhagens e seus acasalamentos, gerando também a visibilidade financeira no mercado equino (BERTOZZO et al., 2014). A inseminação artificial foi uma das biotécnicas precedentes a se destacar e posteriormente, a transferência de embriões, que se sobressai como a mais promissora, sendo o Brasil um dos maiores dirigentes da reprodução equina (LOSINNO e UROSEVIC, 2015).

A transferência de embrião (TE) é uma prática que visa coletar do corpo uterino de uma égua um embrião entre seis e oito dias subsequentes à ovulação, por meio da lavagem uterina de uma equina doadora, possibilitando gerar mais de uma progênie durante o ano, sem afetar suas atividades no decorrer da vida (Pinto et al., 2017). Dentre as vantagens de se utilizar a TE destaca-se a precocidade de fêmeas a vida reprodutiva, maximiza a comercialização de equinos com potencial genético mais específico, bem como a utilização de éguas com idade superior ou com problemas a nível reprodutivo (BETTENCOURT et al., 2018).

Uma parte importante do processo de TE é a sincronização de ambas as éguas, devem estar em condições adequadas, sem presença de alterações uterinas ou ovarianas, e sem problemas relatados de gestação anteriores na receptora, o ciclo das éguas são divididas em duas etapas que geralmente acontecem no decorrer de 21 dias (ARISTIZÁBAL, 2017).

É crucial a execução da sincronização do período fértil e do amadurecimento folicular em éguas nas transferências de embriões, quando o folículo dominante da égua receptora se desprende do ovário, podendo acontecer um dia antes ou dois dias depois da ovulação da doadora. Existem aspectos básicos como por exemplo a seleção da fêmea 
receptora; a utilização de machos de alta qualidade; controle sanitário e manejo adequado que são fundamentais para o resultado da TE (MAIA et al., 2019).

O exame de ultrassom e palpação retal são usados para acompanhar o momento da ovulação e o desenvolvimento folicular nas éguas sincronizadas, e mesmo se sincronizada, as éguas podem ovular com até 3 (três) dias de intervalo (SILVA, 2018).

A realização da coleta no lúmen uterino é parcialmente fácil, podendo ser realizada a partir do sexto dia e se estender no sétimo, oitavo até o nono dia depois da ovulação, sendo que o embrião se estabelece no corpo uterino da égua com 5 ou 6 dias de estágio (BETTENCOURT et al., 2018). Os embriões encontrados no lavado uterino são levados para placa de Petri, que serão subestimados a uma avaliação morfológica e denominados viáveis podendo ser inoculados em receptoras pela técnica não cirúrgica (MONTECHIESI, 2015).

Após o embrião ser transferido para a receptora, a gestação é diagnosticada através da realização do exame ultrassonográfico, pela identificação de uma vesícula de 10 a 15 milímetros, próximo de uma extremidade do útero, em torno de 14 dias seguidos da ovulação, com forma arredondada, com líquido no seu interior, aparecendo em preto no vídeo. A forma esférica da vesícula embrionária, característica desta espécie, possibilita sua fácil identificação no interior do útero, quando atingem um determinado diâmetro, podendo ser observado através da utilização da ultrassonografia obtendo resultado antecipado de gestação (MASKO et al., 2018).

O objetivo deste estudo foi observar a quantidade de embriões coletados e o número de prenhezes confirmadas aplicando-se a técnica de transferência de embrião no município de Mojuí dos Campos, no Baixo Amazonas, Pará.

\section{MATERIAL E MÉTODOS}

\section{Local do estudo}

O estudo foi realizado em uma propriedade rural do município de Mojuí dos Campos, localizado na Microrregião de Santarém, pertencente a Mesorregião do Baixo Amazonas, no estado do Pará - Amazônia Oriental.

\section{Animais e design experimental}


Durante os anos de 2018 e 2019, foram utilizadas como doadoras e receptoras, respectivamente, 5 e 25 éguas mestiças da raça quarto de milha - em diferentes graus de sangue, para que fossem alcançadas 10 transferências de embriões. Os animais apresentavam-se clinicamente saudáveis e não estavam em lactação. A idade variou entre 6 e 10 anos e o escore de condição corporal entre 4 e 5, segundo a Escala de Henneke, com pesos entre 350 e $450 \mathrm{~kg}$ para cada indivíduo (HENNEKE et al., 1983).

Os animais foram acompanhados por meio de ultrassonografia por via transretal, com aparelho modelo Mindray, DP-50Vet, equipado com transdutor linear de $5.0 \mathrm{MHz}$, de onde se obtiveram dados sobre desenvolvimento de folículos ovarianos e tônus do útero. Os folículos foram medidos individualmente para o controle simultâneo entre as éguas.

Quando o folículo dominante das éguas concedentes alcançava $35 \mathrm{~mm}$ o garanhão dava início ao protocolo da monta natural assistida, que era realizada uma vez ao dia, em dias alternados, desde que fosse observado o pico ovulatório da égua.

Concomitantemente era avaliado o amadurecimento de um folículo dominante das receptoras para ovularem até três dias depois da doadora. Sempre que necessário, melhor dizendo, nos casos que as receptoras não estavam em sincronia com a doadora, utilizavase deslorelina, análogo do GnRH (hormônio liberador de gonadotrofina), chamado de Sincrorrelin ${ }^{\circledR}$ equivalente a $3 \mathrm{ml}$, por via intramuscular, mantendo-se o acompanhamento folicular das receptoras até a observação da ovulação.

\section{Cobertura e coleta do embrião}

Foram selecionados 2 garanhões da raça quarto de milha, de fertilidade comprovada, nos quais foram exame andrológico antes do início da estação de monta. A seleção ocorreu com base na qualidade genética para a raça, aliado ao histórico de alta libido e taxa positiva de prenhez na propriedade. As coberturas foram realizadas através da monta natural assistida, realizada de acordo com as instruções de Serviço (2018).

Depois da monta assistida, o acompanhamento da atividade folicular continuou diariamente para observar a ovulação da doadora. Após esta ovulação, esperava-se entre 6 e 8 dias para realizar a coleta do embrião, as quais foram realizadas por meio de lavagens uterinas transcervicais, conforme descrito por McKinnon e Squires (1988).

Caso o embrião não fosse observado na primeira lavagem, repetia-se o processo por mais duas vezes. Ao final dos procedimentos nas doadoras, todas receberam 7,5mg 
de Dinoprost Trometamina - PGF2 $\alpha$ (Lutalyse ${ }^{\circledR}$ Zoetis-Pfizer) para promover o encurtamento do diestro e induzir um novo ciclo estral.

Em seguida, procedeu-se à classificação da qualidade do embrião, melhor dizendo, grau I a IV; conforme as recomendações de McCue e Squires (2015), para que não fossem utilizados embriões degenerados ou mortos.

Para se atingir 10 coletas de embriões foram utilizadas 5 doadoras, refiram-se, D1, D2, D3, D4 e D5. Foram priorizadas para receber o embrião, àquelas receptoras que tivessem ovulado há pelo menos 6 dias da coleta do embrião.

Após os procedimentos, as éguas doadoras eram mantidas em baias e as éguas receptoras eram soltas a campo para aguardar a confirmação de prenhez que ocorria por meio de avaliação ultrassonográfica nos dias 15 e 25, pós transferência.

\section{Análise dos dados}

Os dados foram tabulados e organizados em planilhas do Microsoft excel ${ }^{\circledR} 2016$, sendo expostos os dados percentuais sempre que possível.

\section{RESULTADOS}

Foram coletados 10 embriões por meio da técnica utilizada. Para isso, foram utilizadas 5 doadoras, refiram-se, D1, D2, D3, D4 e D5 das quais foram colhidos 1, 4, 1, 3 e 1, embriões; respectivamente. Nesta pesquisa, optou-se por realizar até 3 lavagens para cada doadora para que se obtivesse o embrião e por esse motivo, não foi mensurada a taxa de recuperação embrionária por animal.

Os embriões coletados foram transferidos para as receptoras, totalizando 10 TEs. Destas, $80 \%$ apresentaram prenhez positiva aos 15 dias e mantiveram esse resultado por ocasião da confirmação da gestação, aos 25 dias, levando a prenhez até o nascimento do potro. As 2 perdas gestacionais ocorreram nos embriões provenientes das doadoras que forneceram maior número de embriões, quais sejam D2 e D4, respectivamente.

Na Figura 1 é possível observar um embrião de 8 dias, classificado como grau 1, classificado em relação ao estágio como blastocisto expandido.

Figuras 1. Embrião equino com 8 dias, observado em imagem microscópica na objetiva de 40X, Santarém, Pará, Brasil, 2021. 


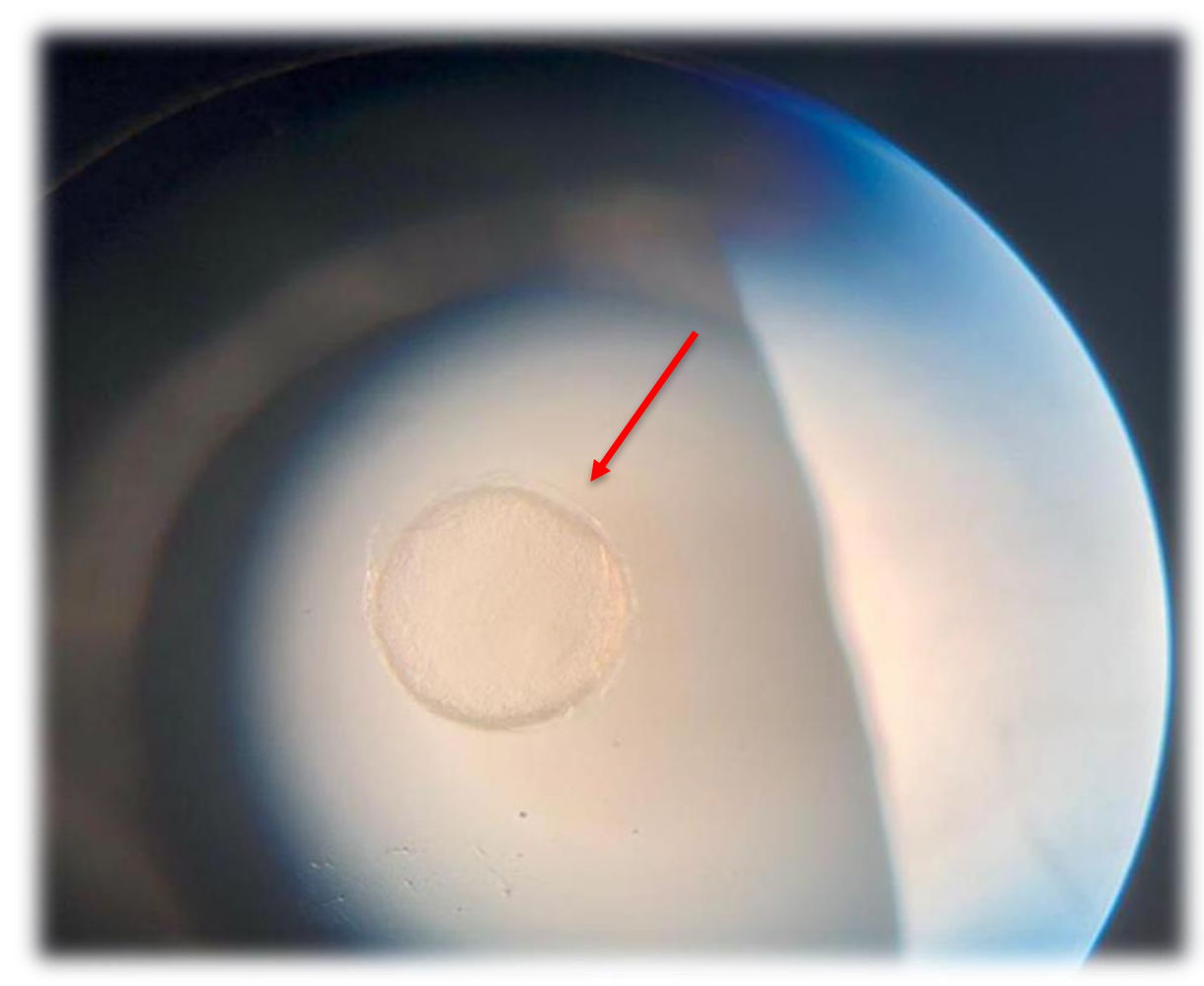

Fonte: Os autores, 2021.

\section{DISCUSSÃO}

Neste estudo, o método padrão utilizado na realização da coleta foi o procedimento não cirúrgico de lavagem uterina. Segundo Mccue e Squires (2015), esse método permite a coleta do embrião de forma mais adequada, sendo a menos invasiva, rápida e a que tem maior probabilidade de confirmação de prenhez.

Segundo Silva et al. (2018), diversos fatores podem atuar sobre os resultados da transferência dos embriões, como alterações climáticas, diferença genética entre a doadora e o garanhão (CAMPBELL, 2014; RUA et al., 2018), idade e o diâmetro da circunferência embrionária (PANZANI et al., 2016).

Alguns desses fatores podem ter contribuído para que duas das receptoras, não tenham conseguido emprenhar. Neste estudo, os animais foram sincronizados para que a transferências dos embriões fosse possibilitada, pois Lopes (2015) descreve que importante a sincronização do período reprodutivo das éguas que são selecionadas para o desempenho do programa de transferência embrionária, usando hormônios exógenos, sendo fundamental a elaboração de um programa estratégico e preparativo para a realização do projeto 
No entanto, diversas vezes a sincronização não alcança o resultado esperado, e para precaver perdas embrionárias, segundo Recalde et al. (2017), é recomendado realizar a sincronização de duas ou mais éguas receptoras correspondente a uma doadora, levando-se em conta, dentre outros fatores, a recomendação de utilizar receptoras que já passaram por outras gestações.

A escolha das fêmeas e dos machos utilizados neste estudo foi baseada em critérios diferentes, que envolvem desde a sanidade, a qualidade espermática ou uterina, até o escore corporal, sendo este um dos principais pontos para o sucesso da TE em equinos (BASTIAON, 2017).

Os resultados de prenhez obtidos neste estudo se aproximam de alguns exemplos da literatura, que variam entre 50 e 75\% (RAZ et al., 2011; BOTELHO et al., 2015), concordando com a ideia apresentada pelo autor, que a realização da técnica seja eficaz.

\section{CONCLUSÃO}

Todas as doadoras responderam ao protocolo testado e apresentaram pelo menos 1 embrião recuperado, sendo que do tal destes, apenas $20 \%$ não se confirmaram como prenhez positiva. Estes resultados apontam como adequada para o local onde foi realizado o experimento, a seleção, a técnica e o manejo utilizados nos animais.

\section{REFERENCIAS}

VALLEJO ARISTIZÁBAL, V. H.; MOGOLLÓN GARCÍA, H. D.; DA SILVA, M.; SANT'ANNA, E.; ANTÔNIO DELL'AQUA JUNIOR; J. Transferência de embriões em éguas receptoras anovulatórias. Revista de Medicina Veterinaria; n. 33; p. 137-147; 2017.

BATISTON, N. R. Modelo de Avaliação Genética e Tendências Fenotípicas para o Desempenho de Cavalos da Raça Quarto de Milha em Provas de Corrida no Brasil. Trabalho de Conclusão de Curso apresentado ao Curso de Bacharelado em Zootecnia, da Universidade Federal de São João Del Rei, Minas Gerais, 2017

BETTENCOURT, E. M. V.; ANTUNES, L.; GONÇALVES, A. R.; BRANCO, S.; ROCHA, A. Reprodução em equinos: Manual Prático. 2018.

BERTOZZO, B. R.; SAMPAIO, B. F. B.; BENDER, É. S. C.; PAGNONCELLI, R. R.; SILVA, E. V. D. C.; ZUCCARI, C. E. S. N. Vantagens e desafios das biotécnicas 
avançadas utilizadas na reprodução equina assistida. Boletim de Indústria Animal, p. 84-93, 2014.

BOTELHO, H. H. V.; PESSOA, G. O.; ROCHA, L. G. P.; YESTE, M. Hormone supplementation protocol using estradiol benzoate and long-acting progesterone is efficient in maintaining pregnancy of anovulatory recipient mares during autumn transitional phase. Animal Reproduction Science, v. 153, p. 39-43, 2015.

CAMPBELL, M. L. H. Embryo transfer in competition horses: Managing mares and expectations. Equine veterinary education, v. 26, n. 6, p. 322-327, 2014.

LOSINNO, L.; UROSEVIC, I. M. Equine embryo transfer. Technical and practical considerations for application on horse production programs. Proceedings...19th International Congress on Biotechnology in Animal Reproduction (ICBAR). Novi Sad, Serbia, p. 23-30, 2015.

LOPES, E. P. Transferência de embriões equinos: maximizando resultados com a escolha de receptoras. Revista Brasileira de Reprodução Animal. v. 39, n. 1, p. 223-229, 2015.

MAIA, H. G. O.; OLIVEIRA, N. J. F.; CROCOMO, L. F. Fisiologia e fatores interferentes na reprodução de éguas. Ciência Animal, v. 29, n. 4, p. 112-123, 2019.

Masko M, Domino M, Skierbiszewska K, Zdrojkowski L, Jasinski, T, Gajewski Z. Monitoring of the mare during the perinatal period at the clinic and in the stable. Equine Vet Edu, doi: 10.1111/eve.13018, 2018.

MCCUE, P. M.; SQUIRES, E. L. Equine Embryo Transfer. 1 Ed., Lexington, Kentucky, USA, Teton Newmedia, 2015.

MCKINNON, A O.; SQUIRES, E. L. Equine embryo transfer. The Veterinary clinics of North America. Equine practice vol. 4,2 (1988): 305-333. doi:10.1016/s07490739(17)30643-0. Diponível em <https://www.sciencedirect.com/science/article/pii/S0749073917306430>. Acessado em 16 nov. 2021.

MONTECHIESI, D. F. Transferência de embriões em equinos e os fatores relacionados as taxas de prenhez. Ciência Animal, v. 25, n. 1, p. 187-194, 2015.

PANZANI, D.; DI VITA, M.; LAINÉ, A.; GUILLAUME, D.; ROTA, A.; TESI, M.; VANNOZZI, I.; CAMILLO, F. Corpus luteum vascularization and progesterone production in autumn and winter cycles of the mare: relationship between ultrasonographic characteristics of corpora lutea and plasma progesterone concentration in the last cycles before anestrus. Journal of equine veterinary science, v. 56, p. 35-39, 2017.

PINTO, M. R.; MIRAGAYA, M. H.; BURNS, P.; DOUGLAS, R.; NEILD, D. M. Strategies for Increasing Reproductive Efficiency in a Commercial Embryo Transfer Program With High Performance Donor Mares Under Training. Journal Equine Veterinary Science, v. 54, p. 93-97, 2017.

RAZ, T.; GREEN, G. M.; CARLEY, S. D.; CARD, C. E. Folliculogenesis: embryo parameters and post-transfer recipient pregnancy rate following equine follicle- 
stimulating hormone (eFSH) treatment in cycling donor mares. Australian Veterinary Journal, v. 89, p. 138-142, 2011.

RECALDE, E.C.S.; SILVA, J.C.; GUIMARÃES, C.F.; VERAS, M.M.M.; BARBOSA, R.G.; ALONSO, M.A.; FERNANDES, C.B. Pluriparidade nas éguas: relação com características maternas, placentárias e neonatais. Ciência Animal Brasileira, v.18, n.1, p.1- 11, 2017.

RUA, M. A. S.; QUIRINO, C. R.; RODRIGUES, A. C. C.; CHRISTO, M. M.; BARRETO, M. A. P. Evaluation of environmental effects on reproductive characteristics of Mangalarga Marchador mares in a commercial embryo transfer program. Animal reproduction science, v. 195, p. 131-138, 2018.

Serviço Nacional de Aprendizagem Rural. Equideocultura: manejo e alimentação. / Serviço Nacional de Aprendizagem Rural. - Brasília: Senar, 2018. 120 p,; il. - (Coleção SENAR, 185), p. 99-102. Disponível em < https://www.cnabrasil.org.br/assets/arquivos/185-EQUIDEOS.pdf>. Acessado em 16 nov. 2021.

SILVA, N. J. Relatório do estágio supervisionado obrigatório (ESO): Atividades desenvolvidas em reprodução equina. 2018. 35 f. Trabalho de Conclusão de Curso (Bacharelado em Medicina Veterinária). Universidade Federal Rural de Pernambuco, Recife, 2018.

SILVA, P. C. A. et al. Taxa de recuperação e características morfológicas de embriões muares (Equus caballus x Equus asinus). Pesquisa Veterinária Brasileira [online]. 2018, v. 38, n. 07 [Acessado 12 Novembro 2021] , pp. 1453-1457. Disponível em: <https://doi.org/10.1590/1678-5150-PVB-4651>. ISSN 1678-5150. https://doi.org/10.1590/1678-5150-PVB-4651.

HENNEKE, D. R.; POTTER G.D.; KREIDER, J. L.; YEATS, B. F. Relationship between condition score, physical measurements and body fat percentage in mares. Equine Veterinary Journal, Cambridge, v.15, n.4. p 371-372, 1983.

MCCUE, P. M.,2011. Transferência de Embriões em Equinos- Avaliação do Embrião. Revista de Educação Continuada em Medicina Veterinária e Zootecnia do CRMVSP / Journal of Continuing Education in Animal Science of CRMV-SP, v. 9, n. 3 (2011), p. 80-83. 